\title{
Bayesian Model Combination and Its Application to Cervical Cancer Detection
}

\author{
Miriam Martínez ${ }^{1}$, Luis Enrique Sucar ${ }^{2}$, Hector Gabriel Acosta $^{3}$, \\ and Nicandro $\mathrm{Cruz}^{3}$ \\ ${ }^{1}$ Tecnológico de Acapulco, Acapulco, Guerrero, México \\ miriamma_ds@hotmail.com \\ ${ }^{2}$ INAOE, Tonantzintla, Puebla, México \\ esucar@inaoep.mx \\ ${ }^{3}$ Universidad Veracruzana, Xalapa, Veracruz, México \\ $\{$ heacosta, ncruz\}@uv.mx
}

\begin{abstract}
We have developed a novel methodology to combine several models using a Bayesian approach. The method selects the most relevant attributes from several models, and produces a Bayesian classifier which has a higher classification rate than any of them, and at the same time is very efficient. Based on conditional information measures, the method eliminates irrelevant variables, and joins or eliminates dependent variables; until an optimal Bayesian classifier is obtained. We have applied this method for diagnosis of precursor lesions of cervical cancer. The temporal evolution of the color changes in a sequence of colposcopy images is analyzed, and the resulting curve is fit to an approximate model. In previous work we develop 3 different mathematical models to describe the temporal evolution of each image region, and based on each model to detect regions that could have cancer. In this paper we combine the three models using our methodology and show very high accurracy for cancer detection, superior to any of the 3 original models.
\end{abstract}

\section{Introduction}

In this work we propose a method to combine several models using a Bayesian approach. The method selects the most relevant attributes from several models, and produces a Bayesian classifier that has a high accuracy (improving all the original models), and at the same time is very efficient. The method includes two phases, discretization and structural improvement. Discretization is based on the minimum description length (MDL) principle, so the number of intervals that minimizes the MDL is obtained per attribute. To deal with dependent and irrelevant attributes, we apply a structural improvement method that eliminates and/or joins attributes, based on mutual and conditional information measures.

We have applied this method for the analysis of colposcopy images for diagnosis of cervical cancer. For this we used the parameters from three mathematical models that characterize the temporal evolution of each pixel in the image. Each model has different number of parameters, in total 11 attributes, all continuous. There are three classes. Our method produces a very simple and efficient classifier with an accuracy of $95 \%$. 
The rest of the document is organized as follows. Section 2 describes the Bayesian classifier and related work in structural improvement. Section 3 presents our method for model combination. Section 4 introduces the cervical cancer detection problem, and Section 5 the image processing and modeling stages. In the next section we present the experimental results. We conclude with a summary and directions for future work.

\section{Related Work}

There are several ways to combine several models for classification purposes. One is to build several classifiers, one for each model, and then combine the outputs of each classifier by, for example, majority voting [15]. Another is to build an ensemble or cascade of classifiers, as in Boosting [7. We propose a different approach. We combine the parameters of all the models into a single classifier, and then use this for classification. We use is a simple, naive Bayes classifier. For building this classifier, we eliminate or join irrelevant and dependent attributes, through a structural improvement stage. Next, we briefly review the naive Bayes classifier and related work in structural improvement.

A Bayesian classifier obtains the posterior probability of each class, $\mathrm{Ci}$, using Bayes rule, as the product of the prior probability of the class by the conditional probability of the attributes given the class (likelihood), divided by the probability of the attributes. The naive Bayes classifier (NBC) makes the simplifying assumption that the attributes are independent given the class, so the likelihood can be obtained by the product of the individual conditional probabilities of each attribute given the class. In this way, the number of parameters increases linearly with the number of attributes, instead of exponentially. Graphically, a NBC can be represented as star-structured Bayesian network [12, with a root node, $C$, that corresponds to the class variable, which is connected to the attributes, $E 1, \ldots, E n$. The attributes are assumed conditionally independent given the class, so there are not arcs between them. Thus, the posterior probability, $P(C i \mid E 1, \ldots, E n)$, of class $C i$ is given by:

$$
P(C i \mid E 1, \ldots, E n)=P(C i) P(E 1 \mid C i) \ldots P(E n \mid C i) / P(E)
$$

\subsection{Structural Improvement}

The naive Bayes classifier assumes that the attribute are independent given the class. If this is not true, there are two basic alternatives. One is to transform the structure of the classifier to a Bayesian network, by introducing directed arcs between the dependent attributes. There are several variants of this approach which are described in 8 . The disadvantage is that the simplicity of the NBC is lost, so learning the model and then classifying new instances becomes more complex. The other alternative is to transform the structure but maintaining a star or tree-structured network. For this, 13 introduces 3 basic operations: (i) eliminate an attribute, (ii) join two attributes into a new combined variable, 


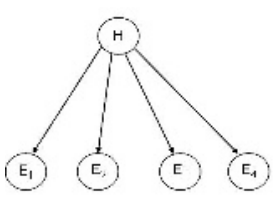

(a) Init al structure

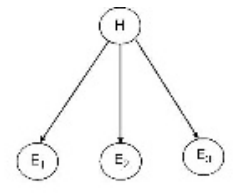

(b) Elimination of $\mathrm{E}$.

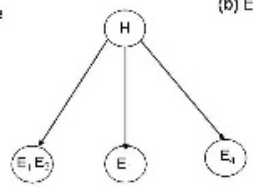

(c) $\sin E, E_{\text {; }}$

Fig. 1. Structural improvement: (a) original structure, (b) one attribute is eliminated, and (c) two attributes are joined into one variable

(iii) introduce a new attribute that makes two dependent attributes independent (hidden node). Figure 1 illustrates the first two operators, which are the ones we use in this work. These operations are based on statistical tests to measure the correlation of pairs of attributes given the class variable. Later, [1] proposes an alternative algorithm for variable elimination and merging (that correspond to the first two operators). The algorithm is based on two search procedures: (i) forward sequential selection and joining (FSSJ) and (ii) backward sequential elimination and joining (BSEJ). This procedures start from a full (empty) structure, and the they select attribute for elimination (addition) or for combination, testing the classification accuracy after each operation. The advantage of these approaches is that they preserve the simplicity and efficiency of the NBC.

In contrast to previous work, we combine elimination of irrelevant attributes with elimination or combination of dependent attributes. Also, our method is based in simple information measures, that avoid testing the classifier for each possible structure, and makes learning more efficient. Finally, our method also integrates a discretization stage to deal with continuous attributes.

\section{Model Combination}

The proposed method obtains an efficient Bayesian classifier for combining several models for classification. It considers (i) discretization of continuous variables, (ii) selection of relevant attributes, and (iii) elimination or combination of dependent attributes. The method obtains a naive Bayes classifier with the minimum number of attributes obtained form the different models. The general algorithm is the following: (1) Initialization, (2) Discretization, (3) Structural Improvement, and (4) Evaluation. Next we describe each stage in detail.

\subsection{Initialization}

This is step is done only once to build the initial classifier. It considers all the attributes from each model (full structure) and an initial partition for the 
continuous attributes with two equal size intervals. Based on this initial structure, the parameters are learned from training data.

\subsection{Discretization}

Given the current structure, in this stage the discretization for each continuous attribute is optimized. The method is based on the minimum description length (MDL) principle. The MDL measure makes a compromise between the accuracy and complexity of the discretization. The measure we use, estimates the accuracy by measuring the mutual information between each attribute and the class; and the complexity by counting the number of parameters. This is done for all the continuous attributes. This stage is described in detail in [10].

\subsection{Structural Improvement}

Given the current discretization (the one with the best MDL from the previous stage), in this phase the structure is improved to eliminate superfluous attributes and eliminate or combine dependent attributes. This phase considers the following stages:

1. The mutual information between each attribute and the class are obtained, and those attributes that do not provide information (below a threshold) are eliminated.

2. The reminding attributes are tested using conditional mutual information (CMI) for each pair of attributes given the class. If this value is high for a pair of attributes it is an indication that the attributes are not independent, so these are candidates for elimination or combination.

3. Each pair of attributes with high CMI are considered for elimination or combination. One is deleted or both are combined into a single attribute. The option with better classification rate is selected.

This process is repeated until there are no more superfluous or dependent attributes. At this stage, the method can go back the discretization stage to optimize again the partitions for each continuous attribute, and then repeat structural improvement.

\subsection{Evaluation}

The final stage consists on evaluating the accuracy of the final classifier with test data.

\section{Cervical Cancer Detection}

Cervical cancer is the first cause of death in Mexican women. If it is detected early, the probability of cure is very high. After a Pap smear test, colposcopy is the most common technique to diagnose this disease because, although it 

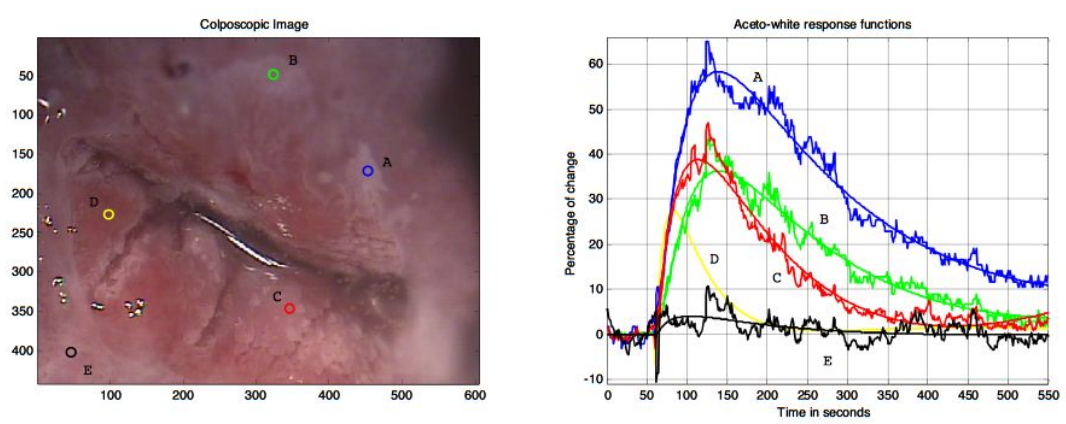

Fig. 2. An example of a colposcopic image, where five regions (A, B, C, D, E) were selected from the image sequence (left). The corresponding Acetowhite response functions are plotted against time (the $\mathrm{X}$-axis is time and the $\mathrm{Y}$-axis is the relative intensity), including the corresponding adjusted curve using model 2 (right).

is more expensive, it has a higher sensitivity and specificity [5]. Basically, the colposcopic test consists of the evaluation of the level of "white" color intensity that the cervical tissue reaches after acetic acid application. An example of a colposcopic image is shown in figure 2 .

There are two problems to develop a colposcopic test. Firstly, the visual analysis has to be done by a well-trained gynecologist. Secondly, the evaluation of the images is subjective in the sense that there are not standard criterion to correlate the tissue color (whitening) with the lesion degree [6]. Many approaches have been proposed to automatically characterize cervical lesions from colposcopic images, most of them use color, texture, or shading, but none of these approaches have shown to be robust enough to be used as a diagnostic tool 149. More recently, some researchers have suggested to use the temporal patterns intrinsic to the color changes, but there is not a complete understanding of how to represent the dynamics of the whitening that occurs after acetic acid application, and how to use it to make an automatic diagnosis adviser [2 4|3]. In previous work [1], we developed a technique to model the temporal evolution of the light changes in the tissue, and to parameterize the dynamics of these temporal changes using different mathematical models. In the following section, this technique is explained, as well as the different mathematical models developed. Then we present how the models are combined based on our methodology.

\section{Image Processing and Modeling}

\subsection{Data Acquisition}

Images were acquired using a colposcope (dfv Vasconsellos model CP-M7, with magnification $16 \mathrm{X}$ without any optical filter) and a color camera (Sony SSCDC50A), with a viewing distance of $20 \mathrm{~cm}$. During the first minute of image acquisition, 60 images $(640 \times 480)$ were taken as base line reference ( 1 frame/second). 
Then, after acetic acid application, five hundred and forty images were taken in 9 minutes using the same sampling frequency. In order to simplify image analysis, the images were processed in gray scale at a resolution of $74 \times 99$.

\subsection{Image Analysis}

The methodology used to analyze colposcopic images involves 3 main stages: (i) image Registration, (ii) time series construction, and (iii) modeling.

The acquisition of colposcopic images spans in average 9 minutes and even though that the patient is fixed, some small random movements are unavoidable, which are often local (patients breathing, movements due to the muscle tonus, etc.). To be able to analyze the sequence of images, i.e. compare and evaluate corresponding structures, the objects in the images should be brought into the same position by removing the differences due to the patient movements - the time series of colposcopic image has to be registered. In our case, we supposed the main source of the misalignments can be modeled by simple translation. The analysis of preliminary registration results showed that this assumption was correct. The method can transform the whole data using the same parameters, or can be local, depending on the local variations. It can be based directly on the image intensity values (area-based methods) or can be done using some features computed from the images (feature-based methods). Because colposcopic images do not contain many distinctive details, an area-based method was chosen.

The classical representative of area-based methods is the normalized crosscorrelation. This method exploits matching directly image intensities. The measure of similarity is computed for window pairs from the input and reference images and its maximum is searched [16]. Because the tissue appearance changes over time due to the effect of the acetic acid effect, the searched pattern defined in the window can look different at a different times, so it is not appropriate to define a static reference image. The input and the reference images are updated continuously starting with the first and second images of the sequence respectively, then the input and the reference images are redefined by the second and the third images and so on. The starting points to initialize the search are updated by the last position in which the pattern window was found. This registration strategy allows not only to contend with the fact that the searched pattern changes over time, but also to reduce the spatial space over which to develop the search.

After the registration process, the signal to noise ratio was increased using a spatial low pass filter implemented using a kernel window $(3 \times 3)$. The intensity value of each pixel over time was used to construct a time series, we called this, the Aceto-white response functions (AwRFs). On a similar way, each AwRF was smoothed using a polynomial filter with a polynomial of order 2 and frames size 60. The filtered time series can be plotted against time (in the image sequence), some examples are shown in figure 2

In previous work [1], three different mathematical models were used to model the evolution of each pixel and based on this to diagnose the probability of cancer. 
Model 1 - Important Points. This model considers 3 important points in the curve:

1. $T s$ : the time to reach its maximum value.

2. $T b$ : the maximum value.

3. Tc: the time from the maximum value until the signal returns to its original value.

Model 2 - Polynomial. Polynomial of degree 5 that approximates the signal, with parameters, $P 1, P 2, P 3, P 4, P 5$ :

$$
A w R F(t)=P 1+P 2 t+P 3 / t+P 4 / t^{2}+P 5 / t^{3}
$$

For $0<t<1$, where $t(i)=$ sample $_{i} /$ samples. The model is a function of time, where the start point of the dynamic function is at time $=60$ seconds (the time at which the acetic acid was applied). So if 600 images were acquired on the image sequence, $t(1)$ is equal to 0.1 (60/600). For analytical purposes the AwRFs can be divided in two sets: the upwards part and the downwards part. The first part represents that the signal reaches the maximum level at some time, and the second one represents the dynamics of how the signal goes back from the maximum to the base line. The peak time can be easily computed by solving $A w R F(t)=0$ for $t$. In the same way, the maximum percentage of change reached can be known substituting for this $t$.

Model 3 - Parabola. Parabola approximation with parameters, $P h, P k, P p$. After an analysis of the AwRFs we found that the first part of the signal can be approximated using a parabola. The standard form of the equation of a parabola with vertex $(h, k)$ and directrix $y=k-p$ is expressed as $(x-h) 2=4 p(y-k)$ with respect to the vertical axis. Where the focus lies on the axis, $p$ units from the vertex. Under this representation the percentage of change and the time peak can be seen as the vertex $(h, k)$ of the parabola, and $p$ as an estimator of the speed of change. An example of the parabolic approximation for different curves is depicted in figure 3 .

\section{Experimental Results}

We evaluated our methodology in the classification of different regions of colposcopy videos. Firstly, some image sequences are classified with the help of an expert. Secondly, based on the training cases, the time series are obtained and described using the 3 models presented in the previous section. Finally, the model combination methodology is applied and a Bayesian classifier is generated that combines the 3 models. This combined classifier was tested with other image sequences, different to the ones for training. 


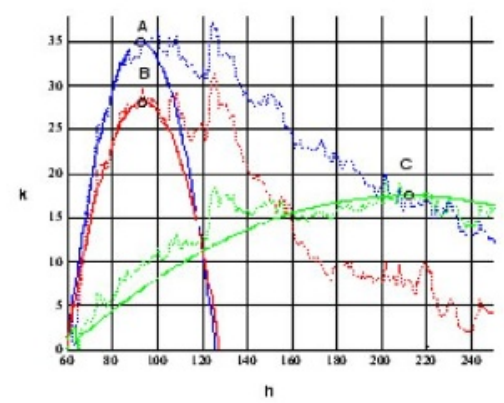

Fig. 3. Some examples of parabolic approximations to typical AwRFs

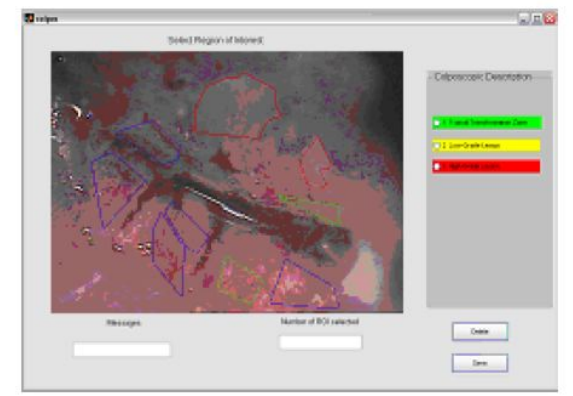

Fig. 4. An example of a colposcopic image with the interface used by the expert to label the training cases

\subsection{Knowledge Acquisition}

The knowledge extraction process was implemented asking the colposcopist to make an analysis of the image sequence. Assisted by a graphical interface in which a representative image of the cervix was shown, the colposcopist was asked to define over the image the different regions with regards to three categories usually reported in a colposcopic description: typical transformation zone, low-grade lesion, and high-grade lesion. The main idea is to ask the expert to make a colposcopic evaluation of one original image and to define regions with an associated label which correspond to a colposcopic feature. This knowledge extraction procedure is made through the use of a graphical interface depicted in figure 4 .

\subsection{Results}

We applied our methodology by considering the 3 models, so all the parameters are the attributes for the classifier, 11 continuous attributes. There are three classes: normal (T), low degree (A) and high degree (B). For the experiments 
we used 1055 sample data labeled by an expert. We used a holdout testing procedure, with approx. $2 / 3$ of the data for training and $1 / 3$ for testing ( 800 for training and 255 for testing).

After obtaining the best discretization for each attribute, the results of the structural improvement stage are summarized in table 1 We can observe that the algorithm eliminates initially a number of redundant attributes, then it combines two dependent attributes and then it eliminates two more attributes, until it arrives at the final structure with 4 attributes: $T s-P k, T c, P 2, P 4$, where one of them is the combination of two attributes of the original models. This final classifier obtains a $95 \%$ accuracy. In comparison, the results with the best single model were of aprox. $90 \%$ accuracy, using the same data and testing procedure. We also compared it with other classifiers using the same data (using all the attributes): (i) naive Bayes, 89\%, (ii) tree augmented naive Bayes (TAN), 94\%, and (iii) decision tree (C4.5), 94\%. The accuracy of our best model is clearly superior to the Naive Bayes and similar to TAN and C4.5. However, the model obtained is much simpler than TAN and C4.5, and thus more efficient.

\section{Conclusions and Future Work}

We have developed a methodology to combine several models using a Bayesian approach. The method selects the most relevant attributes from several models, and produces a Bayesian classifier that has a high accuracy and at the same time is very efficient. Based on conditional information measures, the method eliminates irrelevant variables, and joins or eliminates dependent variables; until an optimal Bayesian classifier is obtained. We have applied this method for diagnosis of precursor lesions of cervical cancer. Based on the dynamics of a video sequence of colposcopy images, 3 different mathematical models were generated to describe the Aceto-white response functions over time. By combining these 3 models with our approach, we generated a Bayesian classifier with 4 attributes (one is a combination of 2 of the original parameters) that produces a $95 \%$ accuracy for the test cases.

We are working on extending this method for dynamic models, based on dynamic Bayesian networks. In the future we will like to apply the methodology

Table 1. Structural improvement stage for cancer diagnosis

\begin{tabular}{|c|c|c|c|}
\hline Operation & No. attr. & attributes & Acc. \\
\hline Join Ts-Pk & 11 & TsTbTcP1P2P3P4P5PhPkPp & 88 \\
Elim. P1 & 10 & Ts-PkTbTcP1P2P3P4P5PhPp & 89 \\
Elim. Pp & 8 & Ts-PkTbTcP2P3P4P5PhPp & 89 \\
Elim. Ph & 7 & Ts-PkTbTcP2P3P4P5Ph & 90 \\
Elim. Tc & 6 & Ts-PkTbTcP2P3P4P5 & 90 \\
Elim. P3 & 5 & Ts-PkTbTcP2P4P5 & 91 \\
Elim. P5 & 4 & Ts-PkTcP2P4P5 & 92 \\
\hline
\end{tabular}


in other domains, considering more models and parameters, to test the scalability of this approach.

\section{References}

1. H. Acosta-Mesa, B. Zitová, H. Ríos-Figueroa, N. Cruz-Ramírez, A. MarínHernández, R. Hernández-Jiménez, B. Cocotle-Ronzón, and E. Hernández-Galicia. Cervical cancer detection using colposcopic images: a temporal approach. In Proc. Sixth International Conference on Computer Science (ENC'05), pages 158-164. IEEE, 2005.

2. M. Anderson, A. M. Jordan J., and F. Sharp. A Text and Atlas of Integrated Colposcopy. Mosby, 1993.

3. W. Pogue B, A. Zelenchuk, W. Harper, G. C. Burke, E. E. Burke, and D. M. Harper. Analysis of acetic acid-induced whitening of high-grade squamous intraepithelial lesions. Journal of Biomedical Optics, pages 397-403, 2001.

4. C. Balas. A novel optical imaging method for the early detection, quantitative grading, and mapping of cancerous and precancerous lesions of cervix. IEEE Transactions on medical imaging, pages 96-104, 2001.

5. E. Burghardt, P. Hellmuth, and F. Girardi. Primary care colposcopy. Thieme, 2004.

6. B. L. Craine, C. J. O'Toole, and Q. Ji. Digital imaging colposcopy: Corrected area measurements using shape-from-shading. IEEE Transactions on medical imaging, pages 1003-1010, 1998.

7. R. Freund and E. Schapire. A short introduction to boosting. Journal of the Japanese Society for Artificial Intelligence, 14:771-780, 1999.

8. N. Friedman, D. Geiger, and M. Goldszmidt. Bayesian network classifiers. Machine Learning, 29:131-163, 1997.

9. Q. Ji and Eric Craine. Texture analysis for classification of cervix lesions. IEEE Transactions on medical imaging, pages 1144-1149, 2000.

10. M. Martínez and L.E. Sucar. Learning an optimal naive bayes classifier. In Proc. Congreso de Investigación y Extensión. ITESM, 2006.

11. Michael J. Pazzani. Searching for atribute dependencies in bayesian classifiers. In preliminary Papers of the Intelligence and Statistics, pages 424-429, 1996.

12. J. Pearl. Probabilistic reasoning in intelligent systems. Morgan Kaufmann, Palo Alto, Calif., U.S.A., 1988.

13. L.E. Sucar, D.F. Gillies, and D.A. Gillies. Objective probabilities in expert systems. Artificial Intelligence, 61:187-208, 1993.

14. A. T. Vlastos, Andres Zuluaga, and Michele Follen. New approaches to cervical cancer screening. Contemporany Ob/Gyn, pages 87-103, 2002.

15. X. Yi, Z. Kuo, and C. Zhang. Classifier combination based on active learning. In Proc. of the 17th International Conference on Pattern Recognition (ICPR'04), pages 184-187. IEEE, 2004.

16. B. Zitova. Image registration methods: a survey. Image and Vision Computing, pages $977-1000,2003$. 